\title{
Correspondence: Spontaneous secondary mutations confound analysis of the essential two-component system WalKR in Staphylococcus aureus
}

\author{
Ian R. Monk', Benjamin P. Howden ${ }^{1}$, Torsten Seemann ${ }^{2} \&$ Timothy P. Stinear ${ }^{1}$
}

Nature Communications 8:14403 doi: 10.1038/ncomms14403 (2017); Published 6 Feb 2017

Ji et al. ${ }^{1}$ recently described the structure of the extracytoplasmic Per-Arnt-Sim (PAS) domain of WalK (WalK ${ }^{\mathrm{EC}-\mathrm{PAS}}$ ), the sensor kinase of the essential two-component system WalKR in Staphylococcus aureus. The authors made two independent walK mutants in $S$. aureus, each with a single amino acid alteration in WalK ${ }^{\mathrm{EC}-\mathrm{PAS}}$, inferring from the structure that these residues might be important for signal transduction. We have also been exploring the function of WalKR and were surprised by the striking phenotypic impacts of these single amino acid substitutions.

The authors showed that their WalK ${ }^{\mathrm{EC}-\mathrm{PAS}}$ mutants (WalK ${ }^{\mathrm{D} 119 \mathrm{~A}}$ and $\mathrm{WalK}^{\mathrm{V} 149 \mathrm{~A}}$ ) caused reduced susceptibility to lysostaphin, loss of sheep blood haemolysis, reduced biofilm formation and reduced virulence compared with parental methicillin-sensitive $S$. aureus strain Newman ${ }^{2}$. RNA-seq comparisons of the two mutants to wild type identified substantial transcriptional changes. Structure-based virtual screening was used to predict that 2,4-dihydroxybenzophenone (DHBP) would interact with WalK ${ }^{\mathrm{EC}-\mathrm{PAS}}$. As DHBP appeared to stimulate lysostaphin-induced lysis and biofilm formation in strain Newman, the authors postulated that it was activating WalKR. They then measured transcriptional responses of Newman after no treatment, DHBP exposure and in their D119A mutant and reported that there were 41 genes that inversely expressed the WalK $\mathrm{K}^{\mathrm{D} 119 \mathrm{~A}}$ mutant compared with DHBP-treated cells, concluding that this supported a role for DHBP in activating WalKR. No direct biochemical evidence of WalKR activation was presented ${ }^{1}$.

To investigate further, we were provided with $S$. aureus Newman wild-type, WalK ${ }^{\mathrm{D} 119 \mathrm{~A}}$ and WalK ${ }^{\mathrm{V} 149 \mathrm{~A}}$ by the senior author, Chuan He, University of Chicago (UoC) ${ }^{1}$. We sequenced the genomes of University of Melbourne (UoM) and UoC Newman strains and compared their sequences with the reference ${ }^{2}$, with the two strains differing by only one synonymous mutation in $\operatorname{sbnF}$ (NWMN_0065). Using allelic exchange, we recreated the WalK ${ }^{\mathrm{D} 119 \mathrm{~A}}$ mutation in the Newman wild type and the USA300 lineage strain NRS384 ${ }^{3}$. We confirmed by genome sequencing that only the $\mathrm{AT} \rightarrow \mathrm{CG}$ substitution in
WalK ${ }^{\text {EC-PAS }}$ was introduced in both strains (NC 009641 chromosome position 25994). We then tested UoM Newman WalK ${ }^{\mathrm{D} 119 \mathrm{~A}}$ and NRS384 WalK ${ }^{\mathrm{D} 119 \mathrm{~A}}$ for the key phenotype changes observed by Ji et al. ${ }^{1}$. However, in contrast to Ji et al., ${ }^{1}$ we observed that UoM WalK ${ }^{\mathrm{Di} 19 \mathrm{~A}}$ and NRS384 WalK ${ }^{\mathrm{D} 119 \mathrm{~A}}$ were fully haemolytic (Fig. 1a) and exhibited identical growth curve kinetics as the parental strains (Fig. 1b). Concurrent screening of UoC Newman WalK ${ }^{\mathrm{D} 119 \mathrm{~A}}$ confirmed that it was non-hemolytic (Fig. 1a). The mutant also grew to an increased $\mathrm{OD}_{600}$, as reported (Fig. 1b) ${ }^{1}$, although CFU were identical to wild type, suggesting that the increase in OD was not due to increased growth. Additionally, UoC WalK ${ }^{\mathrm{D} 119 \mathrm{~A}}$ consistently exhibited larger colonies than Newman or UoM WalK ${ }^{\mathrm{D} 119 \mathrm{~A}}$. We next measured the sensitivity of the strains to lysostaphin by cell viability (Fig. 1c). We observed that the UoC WalK ${ }^{\mathrm{D} 119 \mathrm{~A}}$ mutant was significantly more sensitive (not resistant) to lysostaphin than wild type (3- $\log _{10}$ reduction versus Newman), whereas the UoM WalK ${ }^{\mathrm{D} 119 \mathrm{~A}}$ mutant showed no change. Interestingly, the WalK ${ }^{\mathrm{D} 119 \mathrm{~A}}$ mutation in NRS384 caused an increase in lysostaphin sensitivity, suggesting that the mutation contributes to WalKR activation rather than repression, as proposed by Ji et al. ${ }^{1,4}$.

To resolve the above discrepancies, we subjected UoC WalK ${ }^{\mathrm{D} 119 \mathrm{~A}}$ and $\mathrm{UoC}$ WalK ${ }^{\mathrm{V} 149}$ to whole-genome sequencing. Relative to Newman, and in addition to their expected walk ${ }^{\mathrm{EC}-\mathrm{PAS}}$ changes, both UoC mutants D119A and V149A had acquired four additional mutations (Table 1). Most notably, two independent loss-of-function mutations in saeRS, a major two-component regulator that controls the expression of many genes involved in virulence and biofilm formation ${ }^{5-8}$. The UoC WalK ${ }^{\mathrm{D} 119}$ had a TT insertion at position 757519 that introduced a frameshift to saes. The UoC WalK ${ }^{\mathrm{V} 149 \mathrm{~A}}$ had a $\mathrm{G} \rightarrow \mathrm{T}$ substitution at position 757889 that introduced a premature stop codon to saeR. It is these secondary mutations in saeRS, rather than the targeted mutations in walk ${ }^{\mathrm{EC}-\mathrm{PAS}}$, that likely explain the phenotypes observed by Ji et al. ${ }^{1}$ (reduced biofilm, loss of haemolysis, reduced virulence). We also mapped the authors' RNA-seq reads for WalK ${ }^{\mathrm{D} 119 \mathrm{~A}}$ and WalK ${ }^{\mathrm{V} 149 \mathrm{~A}}$

\footnotetext{
${ }^{1}$ Department of Microbiology and Immunology, Peter Doherty Institute for Infection and Immunity, University of Melbourne, Melbourne, Victoria 3000 , Australia. ${ }^{2}$ Victorian Life Sciences Computation Initiative, University of Melbourne, Carlton, Victoria 3010, Australia. Correspondence and requests for materials should be addressed to T.P.S. (email: tstinear@unimelb.edu.au).
} 
a
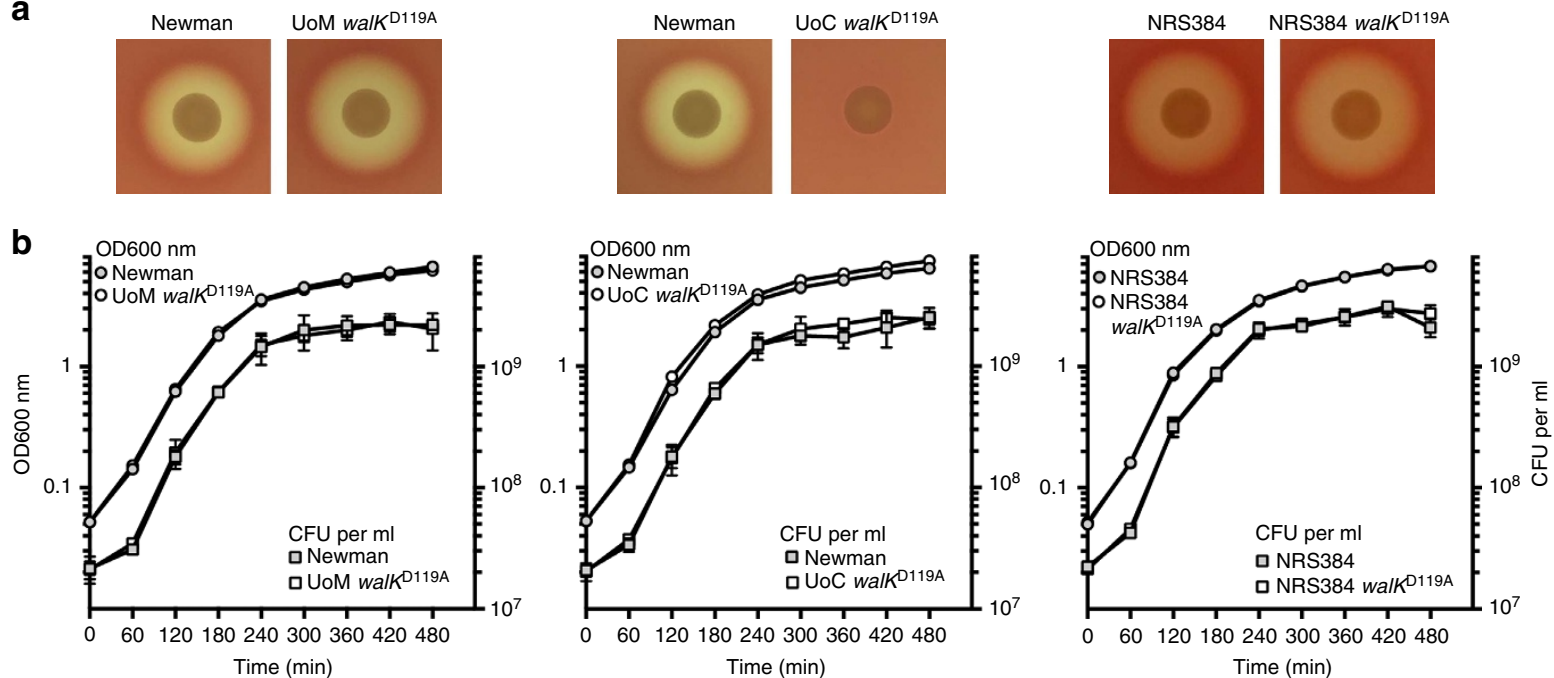

C
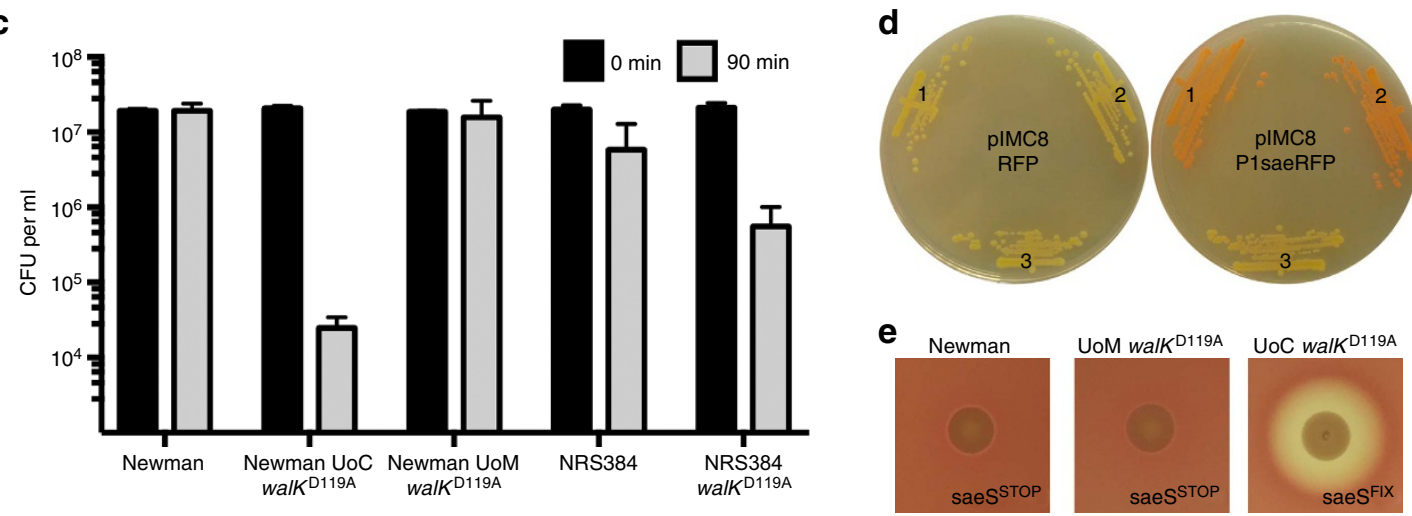

Figure 1 | Phenotypic screening of walK ${ }^{\text {EC-PAS }}$ mutants. (a) No impact on haemolysis was observed for the WalKD119A mutation in either Newman or NRS384 on sheep blood agar. (b) Growth kinetics were identical for the newly created WalK ${ }^{D 119 A}$ mutants in TSB at $37^{\circ} \mathrm{C}$ with aeration (200 r.p.m.) when compared with the parental strain. Optical density $(O)$ and colony-forming units $(\square)$ were enumerated. (c) In a lysostaphin growth sensitivity assay, the UoC WalK ${ }^{\text {D119A }}$ exhibited a loss of viability, while the parent or UoM WalK ${ }^{D 119 A}$ did not. The WalK ${ }^{\text {D119A }}$ mutation in the NRS384 background enhanced sensitivity to lysostaphin when compared with the parental strain. Error bars depict the s.d. of the mean from three independent experiments. (d) P1 sae promoter activity with a DsRED reporter. Left: no promoter. Right: P1 sae driving RFP expression; (1) Newman (2) UoM WalKD119A (3) UoC WalKD119A. No expression of sae was observed in UoC WalK ${ }^{\mathrm{D} 119 \mathrm{~A}}$. (e) By allelic exchange, the UoC saes mutation was introduced into Newman or UoM WalK ${ }^{\mathrm{D} 119 \mathrm{~A}}$ $\left(\right.$ saeS $^{\text {STOP) }}$ ) with haemolysis abolished, while introduction of the wild-type saeS gene into UoC WalK ${ }^{\mathrm{D} 119 \mathrm{~A}}$ (saeS ${ }^{\mathrm{FIX}}$ ) restored haemolysis.

Table 1 | Whole-genome sequencing identified the introduction of unintended polymorphisms within the S. aureus Newman WalK mutants.

\begin{tabular}{|c|c|c|c|c|c|c|}
\hline $\begin{array}{l}\text { Position of } \\
\text { S. aureus Newman } \\
\text { (NC_009641) }\end{array}$ & $\begin{array}{l}\text { UoC Newman } \\
\text { (Acc: } \\
\text { ERR1450026) }\end{array}$ & $\begin{array}{l}\text { UoC Walk } \\
\text { (Acc: } \\
\text { ERR1450027) }\end{array}$ & $\begin{array}{l}\text { UoC WalkV149A } \\
\text { (Acc: } \\
\text { ERR1450028) }\end{array}$ & $\begin{array}{l}\text { Locus_tag } \\
\text { (NWMN_) }\end{array}$ & Gene & $\begin{array}{l}\text { Comment (product, predicted consequence of } \\
\text { mutation) } c .=\text { codon; p. = amino acid position }\end{array}$ \\
\hline 25994 & A & C & A & 0018 & walk & $\begin{array}{l}\text { Sensor kinase: missense_variant c.356A >C } \\
\text { p.Asp119Ala }\end{array}$ \\
\hline 26084 & $\mathrm{~T}$ & $\mathrm{~T}$ & C & 0018 & walk & $\begin{array}{l}\text { Sensor kinase: missense_variant c.446T }>\text { C } \\
\text { p.Val149Ala }\end{array}$ \\
\hline 87710 & A & A & A & 0065 & $s b n F$ & Siderophore biosynthesis: synomous c. $425 G>A$ \\
\hline 757519 & - & $\mathrm{TT}$ & - & 0674 & saes & $\begin{array}{l}\text { Sensor kinase: frameshift variant c.152_153ins AA } \\
\text { p.Thr52fs }\end{array}$ \\
\hline 757889 & C & C & A & 0675 & saeR & $\begin{array}{l}\text { DNA-binding response regulator stop_gained } \\
\text { c.469G > T p.Glu157* }\end{array}$ \\
\hline 820314 & C & $\mathrm{T}$ & $\mathrm{T}$ & 0728 & hprK & $\begin{array}{l}\text { HPr kinase/phosphorylase: missense_variant c.383C } \\
>\text { T p.Ala128Val }\end{array}$ \\
\hline 897870 & - & TG & TG & 0810 & & $\begin{array}{l}\text { Truncated hypothetical. Created full-length gene. } \\
\text { c.98_99ins TG p. 50-119 }\end{array}$ \\
\hline 1914999 & $\mathrm{~T}$ & $\mathrm{~T}$ & C & & & Intragenic between NWMN_1716/1717 \\
\hline 2370632 & C & $\mathrm{G}$ & C & 2142 & $r p / N$ & $\begin{array}{l}50 S \text { ribosomal protein L14: missense_variant } \\
\text { c. } 86 G>\text { C p.Gly29Ala }\end{array}$ \\
\hline
\end{tabular}


(GSE75731) and readily detected the same saeRS mutations. To confirm the predicted functional consequences of the saeRS mutations, we used a P1 Sae red fluorescence reporter plasmid ${ }^{5}$. No fluorescence activity was detected in the UoC WalK ${ }^{\mathrm{D} 119 \mathrm{~A}}$ strain containing the P1 Sae reporter, consistent with the predicted truncation in the histidine kinase preventing phosphorylation of SaeR, whereas high-level expression of P1 Sae from Newman and UoM WalK ${ }^{\mathrm{D} 119 \mathrm{~A}}$ was observed leading to red colonies (Fig. 1d). We then recreated the mutated saeS allele from UoC WalK ${ }^{\mathrm{D} 119 \mathrm{~A}}$ in both wild-type Newman and UoM WalK ${ }^{\mathrm{D} 119 \mathrm{~A}}$ (Fig. 1e). The mutation abolished haemolysis on sheep blood agar. We then repaired the saeS mutation in UoC WalK ${ }^{\mathrm{D} 119 \mathrm{~A}}$ and observed restoration of wild-type haemolysis (Fig. 1e). These results show that the UoC WalK ${ }^{\mathrm{D} 119 \mathrm{~A}}$ strain is an sae mutant with the majority of the phenotypic changes reported in this strain (including the reported RNA-seq changes) likely associated with this mutation rather than $\mathrm{WalK}^{\mathrm{D} 119 \mathrm{~A}}$. The unintended secondary mutations in a major $S$. aureus regulatory locus preclude analysis of the role of WalK ${ }^{\mathrm{EC}-\mathrm{PAS}}$ domain in WalKR signal transduction.

There is precedence for this specific phenomenon. Sun et al. ${ }^{5}$ showed that elevated temperature and antibiotic selection used during the $S$. aureus mutagenesis process can aid in the selection of saeRS mutations. How Ji et al. ${ }^{1}$ managed to complement the mutations (D119A and V149A) by phage integrase plasmid expression (pCL55) of wild-type walKR remains to be explained. We have been unable thus far to obtain the complemented mutants for analysis.

We also observed that UoC WalK ${ }^{\mathrm{D} 119 \mathrm{~A}}$ and $\mathrm{UoC}$ WalK ${ }^{\mathrm{V} 149 \mathrm{~A}}$ exhibited larger colonies compared with wild type, a phenotypic difference not discussed by Ji et al. ${ }^{1}$. This change in both mutants might be explained by the $\mathrm{C} \rightarrow \mathrm{T}$ substitution observed at 820314 , leading to an A128V change in HprK, a serine kinase known to be involved in catabolite repression and associated with a spreading colony phenotype 9 .

Ji et al. ${ }^{1}$ used RNA-seq and the inverse expression profiles of the WalK ${ }^{\mathrm{D} 119 \mathrm{~A}}$ mutant and DHBP treatment of the wild type to 'prove' that DHBP is signaling through WalK ${ }^{\mathrm{EC}-\mathrm{PAS}}$, but this conclusion is confounded by the saeRS mutations. In addition, the authors failed to apply any filter for false discovery rate to their RNA-seq analysis. This analysis without statistical significance thresholds is not meaningful ${ }^{10}$. We also repeated the lysostaphin assay with and without the addition of $75 \mu \mathrm{M}$ DHBP. We failed to observe the reported loss of turbidity in Newman pretreated with DHBP upon lysostaphin treatment ${ }^{1}$ (Supplementary Fig. 1.).

The discovery of small-molecule inhibitors of WalKR function would represent a major advance in the fight against multidrugresistant $S$. aureus. Unfortunately, the presence of unintended saeRS mutations in their walk ${ }^{\mathrm{EC}-\mathrm{PAS}}$ mutants invalidate their conclusions with respect to role of the WalK extracytoplasmic domain in controlling WalKR function. Using a clean D119A mutant, we observed opposing results: with increased sensitivity to lysostaphin, a phenotype previously linked with enhanced activity of $\mathrm{WalKR}^{4}$. In our own WalKR research, we have observed a propensity for mutations introduced into this locus to yield secondary compensatory events ${ }^{11}$. These secondary changes can confound analysis of this essential two-component system and highlight the extreme care needed when manipulating this locus and then attributing specific phenotypes to specific mutational changes.

\section{Methods}

Bacteria and molecular tools. The S. aureus UoM Newman was obtained from Professor Tim Foster (Trinity College Dublin); NRS384 was obtained from BEI resources (www.beiresources.org). S. aureus was routinely grown in Tryptic Soy
Broth (TSB-Oxoid) at $37^{\circ} \mathrm{C}$ with aeration at 200 r.p.m. Primers were purchased from IDT (www. idtdna.com) with primer sequences detailed in Supplementary Table 1. Restriction enzymes, Phusion DNA polymerase and T4 DNA ligase were purchased from New England Biolabs. Genomic DNA was isolated from $1 \mathrm{ml}$ of an overnight culture (DNeasy Blood and Tissue Kit-Qiagen) pretreated with $100 \mu \mathrm{g}$ of lysostaphin (Sigma cat. no. L7386). DHBP was purchased from Sigma (cat. no. $126217 ; 100 \mathrm{~g})$.

Lysostaphin sensitivity assay. Overnight cultures of $S$. aureus were diluted 1:100 in fresh, prewarmed TSB in the presence of $0.2 \mu \mathrm{g} \mathrm{ml}^{-1}$ of lysostaphin with or without $75 \mu \mathrm{M}$ DHBP (100 mM stock in methanol). Broths were incubated statically at $37^{\circ} \mathrm{C}$. Colony-forming units were determined by spot plate dilution on Brain heart infusion agar (Difco) at 0 and $90 \mathrm{~min}$. Limit of detection for the assay was $10^{3} \mathrm{CFU} \mathrm{ml}^{-1}$.

Construction of pIMC8-RFP and SLiCE cloning. The S. aureus codon optimized DsRED red fluorescent protein and upstream TIR sequence from pRFP-F (ref. 12) was PCR amplified with primers IM314/IM315. The product was digested with $\mathrm{KpnI} / \mathrm{SacI}$ and cloned into the complementary digested pIMC8 (non-temperaturesensitive version of pIMC5 (ref. 13)), creating pIMC8-RFP. To clone into pIMAY$\mathrm{Z}^{3}$ and pIMC8-RFP, primers were tailed with $30 \mathrm{nt}$ of complementary sequence to the plasmid. Amplimers were inserted with seamless ligation cloning extract (SLiCE; ref. 14) into the vector (pIMAY-Z: walRK ${ }^{\mathrm{D} 119 \AA}, s^{S T O P}$, sae ${ }^{F I X}$; pIMC8RFP: P1 sae). Either vector was linearized with KpnI, gel extracted and PCR amplified with primers IM1/IM2 (pIMAY-Z) or IM1/IM385 (pIMC8-RFP). Both amplimers (vector and insert) were combined in a $10 \mu l$ reaction containing $1 \times \mathrm{T} 4$ ligase buffer, with $1 \mu \mathrm{l}$ of SLiCE extract. The reaction was incubated at $37^{\circ} \mathrm{C}$ for $1 \mathrm{~h}$ and then transformed into Escherichia coli strain $\mathrm{IM}^{2} 08 \mathrm{~B}^{3}$, with selection on Luria agar plates containing chloramphenicol $10 \mathrm{\mu g} \mathrm{ml}^{-1}$. Plasmids were extracted and directly transformed by electroporation into the target $S$. aureus strain ${ }^{3}$.

Production of SLiCE extract. The SLiCE was isolated from DY380 (ref. 14) grown in $50 \mathrm{ml} 2 \times \mathrm{xYT}(1.6 \%$ Tryptone, $1 \%$ Yeast Extract, $0.5 \% \mathrm{NaCl})$ at $30^{\circ} \mathrm{C}$ after a $1: 100$ dilution of the overnight culture. Once the culture reached an $\mathrm{OD}_{600}$ of $\sim 2.5$, the cells were moved to $42^{\circ} \mathrm{C}$ for $25 \mathrm{~min}$ (addition of $50 \mathrm{ml}$ of $42{ }^{\circ} \mathrm{C} 2 \mathrm{xYT}$ ). Cells were processed as described by Zhang et al. ${ }^{14}$, with the pellet lysed in $500 \mu$ l of Cellytic B cell lysis reagent (C87040; $10 \mathrm{ml}$; Sigma).

Whole-genome sequencing and data analysis. Whole-genome sequencing was performed using the Illumina NextSeq $(2 \times 150 \mathrm{bp}$ chemistry), with library preparation using Nextera XT (Illumina). Resulting reads were mapped to the S. aureus Newman reference (Accession: NC_009641) using Snippy v3.1 (https://github.com/tseemann/snippy). Note that 89 substitutions, 20 deletions and 25 insertions were shared between UoC and UoM Newman strains compared with the NC_009641 reference sequence, representing likely sequencing errors in the 2008 published reference ${ }^{2}$.

Data availability. All sequencing data used in this study have been deposited in the National Center for Biotechnology Information BioProject database and are accessible through the BioProject accession number PRJEB14381 (https://www.ncbi.nlm.nih.gov/bioproject/325902). An updated S. aureus Newman genome sequence is available (Genbank reference: NZ_LT598688.1).

\section{References}

1. Ji, Q. et al. Structure and mechanism of the essential two-component signal-transduction system WalKR in Staphylococcus aureus. Nat. Commun. 7, 11000 (2016)

2. Baba, T., Bae, T., Schneewind, O., Takeuchi, F. \& Hiramatsu, K. Genome sequence of Staphylococcus aureus strain Newman and comparative analysis of staphylococcal genomes: polymorphism and evolution of two major pathogenicity islands. J. Bacteriol. 190, 300-310 (2008).

3. Monk, I. R., Tree, J. J., Howden, B. P., Stinear, T. P. \& Foster, T. J. Complete bypass of restriction systems for major Staphylococcus aureus lineages. mBio 6, e00308-e00315 (2015).

4. Dubrac, S., Boneca, I. G., Poupel, O. \& Msadek, T. New insights into the WalK/WalR (YycG/YycF) essential signal transduction pathway reveal a major role in controlling cell wall metabolism and biofilm formation in Staphylococcus aureus. J. Bacteriol. 189, 8257-8269 (2007).

5. Sun, F. et al. Aureusimines in Staphylococcus aureus are not involved in virulence. PLoS ONE 5, e15703 (2010).

6. Nygaard, T. K. et al. SaeR binds a consensus sequence within virulence gene promoters to advance USA300 pathogenesis. J. Infect. Dis. 201, 241-254 (2010)

7. Liang, X. et al. Inactivation of a two-component signal transduction system, SaeRS, eliminates adherence and attenuates virulence of Staphylococcus aureus. Infect. Immun. 74, 4655-4665 (2006).

8. Cue, D. et al. SaeRS-dependent inhibition of biofilm formation in Staphylococcus aureus Newman. PLoS ONE 10, e0123027 (2015). 
9. Ueda, T., Kaito, C., Omae, Y. \& Sekimizu, K. Sugar-responsive gene expression and the agr system are required for colony spreading in Staphylococcus aureus. Microb. Pathog. 51, 178-185 (2011).

10. Conesa, A. et al. A survey of best practices for RNA-seq data analysis. Genome Biol. 17, 13 (2016).

11. Howden, B. P. et al. Evolution of multidrug resistance during Staphylococcus aureus infection involves mutation of the essential two component regulator WalKR. PLoS Pathog 7, e1002359 (2011).

12. Bose, J. L., Fey, P. D. \& Bayles, K. W. Genetic tools to enhance the study of gene function and regulation in Staphylococcus aureus. Appl. Environ. Microbiol 79, 2218-2224 (2013).

13. Monk, I. R., Shah, I. M., Xu, M., Tan, M. M. \& Foster, T. J. Transforming the untransformable: application of direct transformation to manipulate genetically Staphylococcus aureus and Staphylococcus epidermidis. mBio 3, doi:10.1128/ mBio.00277-11 (2012).

14. Zhang, Y., Werling, U. \& Edelmann, W. SLiCE: a novel bacterial cell extract-based DNA cloning method. Nucleic Acids Res. 40, e55 (2012).

\section{Acknowledgements}

This research was supported by the National Health and Medical Research Council of Australia (GNT1049192)

\section{Author contributions}

I.R.M. and T.P.S. designed the study; I.R.M., T.S. and T.P.S. conducted the bioinformatic analyses; I.R.M., B.P.H. and T.P.S. wrote the paper.

\section{Additional information}

Supplementary Information accompanies this paper at http://www.nature.com/ naturecommunications

Competing financial interests: The authors declare no competing financial interests.

Reprints and permission information is available online at http://npg.nature.com/ reprintsandpermissions/

How to cite this article: Monk, I. R. et al. Correspondence: Spontaneous secondary mutations confound analysis of the essential two component system WalKR in Staphylococcus aureus. Nat. Commun. 8, 14403 doi: 10.1038/ncomms14403 (2017).

Publisher's note: Springer Nature remains neutral with regard to jurisdictional claims in published maps and institutional affiliations.

(c) (i) This work is licensed under a Creative Commons Attribution 4.0 International License. The images or other third party material in this article are included in the article's Creative Commons license, unless indicated otherwise in the credit line; if the material is not included under the Creative Commons license, users will need to obtain permission from the license holder to reproduce the material. To view a copy of this license, visit http://creativecommons.org/licenses/by/4.0/

(C) The Author(s) 2017 